\title{
Antimicrobial Potentials of Leptolyngbya sp. and Its Synergistic Effects With Antibiotics
}

\author{
Mohsen Abazari ${ }^{1}$, Gholamreza Zarrini ${ }^{2,{ }^{*},}$ Iraj Rasooli ${ }^{1}$ \\ ${ }^{1}$ Laboratory of Microbiology, Department of Biology, Faculty of Sciences, University of Shahed, Tehran, IR Iran \\ 2 Laboratory of Microbiology, Department of Biology, Faculty of Natural Sciences, University of Tabriz, Tabriz, IR Iran \\ *Corresponding author: Gholamreza Zarrini, Laboratory of Microbiology, Department of Biology, Faculty of Natural Sciences, University of Tabriz, Tabriz, IR Iran. Tel: +98-4113392346, \\ E-mail:Zarrini@tabrizu.ac.ir.
}

Received: May 26, 2012; Revised: July 17, 2012; Accepted: August 08, 2012

\begin{abstract}
Background: Resistance to antibiotics is a clinical problem. Cyanobacteria have many more antibiotic productions. Nowadays, combinations of antimicrobial compounds are used.

Objectives: The current research studied antimicrobial properties of Leptolyngbya sp.

Materials and Methods: Chloroform extracts of Leptolyngbya sp. were prepared. Antimicrobial effects of these extracts were evaluated singly and in combination with antibiotics against various microorganisms. Growth optimization, antimicrobial activity and their correlations were studied.

Results: Leptolyngbya sp. had antimicrobial activity individually and in synergy with antibiotics against Gram-positive and Gram-negative bacteria. The highest activity of the chloroform extract was on Gram positive strains and its effects on fungi were moderate, but it had a little effect against Gram negative ones. Phosphate salt was the most important factor for growth and antibacterial activity in C. leptolyngbya. Conclusions: Optimization of medium composition and synergistic antimicrobial activity on the cyanobacteria could lead to production of effective antimicrobials.

Keywords: Cyanobacteria spp.; Leptolyngbya sp.; Synergism
\end{abstract}

\section{Background}

Resistance to antibiotics is a ubiquitous relentless clinical problem. New antibiotic development by pharmaceutical sector has aggravated the challenge of widespread resistance and signals a critical need for innovation (1). Cyanobacteria have a wide range of secondary metabolites including antibiotics (2). Researches to identify antimicrobial compounds produced by cyanobacteria, have recently received attention as a new source of novel antimicrobial substances. Ethyl acetate extract of Spirulenaplatensis can inhibit some Gram positive and Gram negative bacteria and Candida albicans (3). Oscillatoria spp. can produce derivatives with antimicrobial activity (4). Nowadays, combinations of antimicrobials are usually used in medicine to broaden antimicrobial spectrum and generate synergistic effects. Drug combinations have potentials for counteracting drug resistance (1). The economical and oligotrophic growth (5-7) of Leptolyngbya sp. form the aim of this research, whereas arriving at new antimicrobial compounds is of prime importance.

The current research studies the antimicrobial effects of oligotrophic cyanobacterium Leptolyngbya sp. independently and in synergy with antibiotics. This taxonomic cluster was originally named as "LPP - group B" by bacteriologists (8), and has been recently supported and accepted by molecular analysts (9). This cyanobacteria is moderately a new thermophilic halophile or halotolerant genus $(10,11)$. The basic and fundamental requirements for cyanobacterial biotechnology are the availability of natural cyanobacterial sources and understanding their ecobiological properties. Therefore, a detailed study of its habitats was made to identify the available cyanobacterial species and subsequently to isolate, purify and establish a culture collection which could be used for further studies (12).

The successful cultivation and growth of cyanobacteria require modification in media composition (9). Several liquid media have been modified for the culture of marine and freshwater species e.g., artificial seawater nutrients III (ASN-III), Marine Medium (MN), and Bluegreen 11 (BG-11) (8). The modification to find a desirable culture related to requirements of cyanobacteria may differ from species to species (13). In the current research, 
the optimum growth conditions of Leptolyngbya sp. were studied. BG-11 medium is a general culture medium for cyanobacteria. BG-11medium was subject to some modifications in Nitrogen, phosphorus, magnesium, iron and copper contents. The results are discussed.

\section{Objectives}

The current study aimed to access new natural antimicrobial compounds; therefore, the current research studied antimicrobial properties of Leptolyngbya sp.

\section{Materials and Methods}

\subsection{Sampling and Culturing}

The unexplored fresh or hot benthic waters in East Azerbaijan province of Iran were the sampling regions. Samples were taken directly by immersion in water of sampling bottles by "grab" or "snap" sampling methods (14). The samples were stored at a temperate range of 10$17^{\circ} \mathrm{C}$ under dark conditions in a storage box in order to decrease metabolism and to avoid the growth interfering factors during sampling and transportation.

\subsection{Growth Conditions}

The samples were cultured batch wise in BG-11 agar and broth media (15) and illuminated at a light intensity of 3500 lux (16 hours light: 8 hours dark) at $22^{\circ} \mathrm{C}$ and shaking at $100 \mathrm{rpm}$. The samples were screened for Leptolyngbya sp. by different purification methods according to the degree of contamination of samples.

\subsection{Molecular Analysis}

DNA extraction with boiling method For PCR test was performed and then was amplified and electrophoresis (16). PCR products were sequenced and aligned with the cited sequences in NCBI.

\subsection{Oligotrophic and Halophilic or Halotolerant Aspects of the Leptolyngbya sp.}

Growth of Leptolyngbya sp. in oligotrophic state was studied in the CHU NO.10 modified medium. The medium contains very low amounts of nutrients and salts and is therefore only suitable for oligotrophic strains. Halophilic or halotolerant properties of Leptolyngbya sp. were tested with van Rijn and Cohen (RC) Medium (17). RC modified media were prepared at $1 / 3$ and $2 / 3$ reduced salt concentrations compared to the original.

\subsection{Antibacterial and Antifungal Bioassay}

\subsubsection{Extract Preparation}

Leptolyngbya sp. strains grown in the liquid medium were harvested at late exponential phase. The biomass was extracted with hexane, methanol, diethyl ether, ethyl acetate, chloroform organic solvents. Aqueous crude extracts were also used.

\subsubsection{Antimicrobial Activity on Test Microorganisms}

The following bacteria and fungi were used as test organisms: Staphylococcus aureus (PTCC 1112), Micrococcus luteus (PTCC 1625), Escherichia coli (PTCC 1329), Erwiniacarotovora (PTCC 1675), Salmonella typhi (PTCC 1609) and Candida kefyr (ATCC 38296).

Minimum inhibitory concentrations (MIC), Minimum bactericidal inhibition (MBC) and minimum fungicidal inhibition (MFC) were assayed by broth dilution methods $(18,19)$. The antibacterial effects of a combination of the chloroform extract, which exhibited the highest antimicrobial activity with antibiotics, were assessed by the checkerboard test and fractional inhibitory concentration (FIC) (20). Where more than one combination resulted in a change in the MICs value of the extract or antibiotic, the FICI was described as the total of the individual FIC values (20).

\subsection{Evaluation of the Effects of BG-11 Medium Com- ponents on the Optimum Growth and Antimicro- bial Activity ofC. Leptolyngbya BG-11 medium com- ponents}

(0.75 mg) MgSO4.7H2O, (36 mg) CaCl2.2H2O, (1500 $\mathrm{mg}$ ) NaNO3, (30 mg) K2HPO4, (20 mg) Na2CO3, (1 mg) Na2EDTA.2H2O, (6 mg) Ferric ammonium citrate, $(1 \mathrm{ml})$ Micronutrient solution (A5+CO)(8), final volume $1000 \mathrm{ml}$ with deionized water and $\mathrm{pH}$ after filtrate $\sim 7.1$.

(A5+CO) components: (286 mg) H3BO3, (181 mg) $\mathrm{MnCl} 2.4 \mathrm{H} 2 \mathrm{O},(22 \mathrm{mg}) \mathrm{ZnSO} 4.7 \mathrm{H} 2 \mathrm{O},(39 \mathrm{mg}) \mathrm{NaMoO} 4.2 \mathrm{H} 2 \mathrm{O}$, (79 mg) CuSO $4.5 \mathrm{H} 2 \mathrm{O}$ in the final volume $100 \mathrm{ml}$ with deionized water. Culture media was prepared in three forms: $0,1.5$ and 2 fold of BG-11 salts. Incubation conditions were as described earlier. After 13 days, the culture media were taken for growth measurement, determination of dry weight and chloroform extracts antibacterial assay.

\subsection{Growth Measurements}

Chlorophyll-a, from each sample was extracted in $80 \%$ acetone and the content of the pigments was determined using the procedure of Myers and Kratz at an absorbance of $663 \mathrm{~nm}$. Specific growth rate was calculated using the formula: $\mu=[\ln (\mathrm{Bf} / \mathrm{Bi})] /$ ti. Where $\mathrm{Bi}$ is the initial chlorophyll-a, and Bf is the chlorophyll-a, at the end of incubation. Productivity was calculated from the formula: $\mathrm{P}=(\mathrm{Xi}-\mathrm{X0}) /$ ti, where $\mathrm{P}=$ productivity (mg L-1day-1), $\mathrm{Xo}=$ initial biomass density (mg L-1), Xi = biomass density at time $\mathrm{i}(\mathrm{mg} \mathrm{L}-1)$ and $\mathrm{ti}=$ time interval $(\mathrm{h})$ between $\mathrm{XO}$ and $\mathrm{Xi}$ (21). 


\subsection{Determination of Dry Weight}

$10 \mathrm{ml}$ from different cultures were washed several times with distilled water to remove soluble salts. The cyanobacteria cells were then dried at $80^{\circ} \mathrm{C}$ for $30 \mathrm{~min}$ and weighed (22).

\section{Results}

\subsection{Isolation}

Overall, 35 samples were isolated out of which only 2 samples were Leptolyngbya spp.

\subsection{Identification}

The 16S rRNA sequences of two isolates from Heravi location of Mehranrud and Bostan Abad underground hot spring were subject to NCBI blast. The former corresponded to Leptolyngbya nodulosa UTEX 2910 with accession number (gi|119367623|EF122600.1), 99\% identity and 0\% gap. The latter corresponded to Leptolyngbya spp. 0418 with accession number (gi|15842033|EU068735.1), 99\% identity and $0 \%$ gap.

\subsection{Growth Ability}

B-110 culture medium is nitrogen limited medium. The isolated strains of Leptolyngbya sp. were grown in the culture medium and therefore may be considered as putative nitrogen fixing species for Leptolyngbya sp. isolates. These strains relieve suitable growth in the CHU-10 modified medium. Bottle-green and glucose pigments in nitrogen limited media, namely BG-110 and CHU-10 modified culture did not occur. Among the different dilutions of saline RC culture medium, the best growth was observed at 1:3 dilution. Available strains have growth ability in the RC 1/3 modified.

\subsection{Antimicrobial Results}

Chloroform extracts compared to other solvents, showed better results in antimicrobial testing. Therefore, further investigation of the antimicrobial activity with chloroform extract was performed. The Screening tests showed that Gram positive bacteria were more susceptible than Gram negative bacteria. The antimicrobial activities are given in Table 1.

Table 1. Antimicrobial Activity of the Chloroform Extracts of Leptolyngbya sp.1 For Bacteria and Leptolyngbya sp. 2 for Fungi Alone and Different Antibiotics Combined.

\begin{tabular}{|c|c|c|c|c|c|c|}
\hline \multirow[t]{2}{*}{ Strains } & \multirow[t]{2}{*}{ Agent } & \multirow{2}{*}{$\begin{array}{l}\text { MIC, } \mu \mathrm{g} / \mathrm{mL} \\
\text { Alone }\end{array}$} & \multirow{2}{*}{$\begin{array}{l}\text { FIC } \\
\text { Combined }\end{array}$} & \multirow[t]{2}{*}{ FICI } & \multirow[t]{2}{*}{ Outcome } & \multirow[t]{2}{*}{ Type } \\
\hline & & & & & & \\
\hline Micrococcus luteus & Leptolyngbya sp. 1 Gentamicin & $80 />80^{a} 1.2$ & $20(0.3)$ & $0.25(0.25)$ & 0.5 & Synergistic \\
\hline Micrococcus luteus & Leptolyngbya sp. Erythromycin & $80 />80(0.3)$ & $20(0.15)$ & $0.25(0.5)$ & 0.75 & Additive \\
\hline Staphylococcus aureus & Leptolyngbya sp. 1 Gentamicin & $40 / 80(0.6)$ & $20(0.15)$ & $0.5(0.25)$ & 0.75 & Additive \\
\hline Staphylococcus aureus & Leptolyngbya sp. 1 Erythromycin & $40 / 80(0.3)$ & $20(0.15)$ & $0.5(0.5)$ & 1 & Additive \\
\hline Salmonella typhi & Leptolyngbya sp. 1 Gentamicin & Resistance (1.2) & $20(0.6)$ & $0(0.5)$ & 0.5 & Synergistic \\
\hline Salmonella typhi & Leptolyngbya sp. 1 Ceftazidime & Resistance (12.5) & $20(6.2)$ & $0(0.5)$ & 0.5 & Synergistic \\
\hline Candida kefyr & $\begin{array}{l}\text { Leptolyngbya sp. } 2 \text { Amphotericin } \\
\text { B }\end{array}$ & $40 / 60(0.6)$ & $20(0.3)$ & $0.5(0.5)$ & 1 & Additive \\
\hline
\end{tabular}

\footnotetext{
$\mathrm{a}$ The fractions are related to the $\mathrm{MBC}$ data of
}

Table 2 shows the values of maximum specific growth rate ( $\mu$ max) and productivity (P312) 13 days after culturing two strains of Leptolyngbya genera at different salt concentrations of BG-11 culture media. Their antibacterial effects were evident against $M$. luteus . Due to the importance of nitrogen, phosphorus, iron, magnesium and copper ions in the NaNO3, K2HPO4, Ferric ammonium citrate, MgSO4, CuSO4 salts, have been studied in the current research ( Table 2 ). With the exception of phosphorus and copper salts, high concentrations of other elements did not lead to increased antibacterial activity. None of the isolated Leptolyngbya strains showed signs of growth at two-fold increased micronutrients solution (A5+CO) ( Table 2 ).

\section{Discussion}

Leptolyngbya strains grew well in the CHU-10 modified medium. The findings were in support of those of
Douterelo et al. (5), Mateo et al. (6) and Perona et al. (7) in which Leptolyngbya sp. was oligotrophic. In the modified $\mathrm{RC}$, saline concentration was approximately equivalent to $34 \mathrm{psu}$. This means that cyanobacteria have ability for growth in the average saline sea waters. These observations are in agreement with those of Abed et al. (10), and Chatchawan et al. (11). According to the antimicrobial test, about $42.85 \%$ of all the interactions were synergistic, while indifferent and antagonistic interactions were not observed. Additive activity was against the Salmonella typhi. Synergistic activity between Leptolyngbya sp.2 and Amphotericin B against C. kefyr shows that this strain possesses antifungal activity. Over all, strain 2 has more antifungal activity than strain 1 . Antibacterial activity of strain 1 is higher than that of strain 2 . Gram positive bacteria were more susceptible than Gram negative bacteria confirming Dixon et al. report (23). 
Table 2. Growth Ability With $\mu$ max, P 312 Tests and Antibacterial Activity Research With Kirby-Bauer Disk Diffusion Method

\begin{tabular}{|c|c|c|c|}
\hline Treatments & $\mu \max$, day $^{\mathrm{a}}$ & P312, mgL $^{\mathrm{a}}$ day $^{\mathrm{a}}$ & Inhibition Zones $^{b}$ \\
\hline BG-11 plus 0.5 fold of K2HPO4 salt to Leptolyngbya sp. 1 & $0.178 \pm 0.004$ & $9.93 \pm 0.16$ & Resistance \\
\hline BG-11plus 1 fold of K2HPO4 salt to Leptolyngbya sp.1 & $0.224 \pm 0.002$ & $10.57 \pm 0.27$ & $9 \pm 0.63$ \\
\hline BG-110plus 1 fold of K2HPO salt to to Leptolyngbya sp.1 & $0.088 \pm 0.003$ & $4.8 \pm 0.75$ & $8 \pm 1.09$ \\
\hline No K2HPO4salt BG-11 to Leptolyngbya sp.1 & No growth & No growth & $14 \pm 0$ \\
\hline BG-11plus 0.5 fold of K2HPO4 salt to Leptolyngbya sp.2 & $0.162 \pm 0.004$ & $6.89 \pm 0.33$ & Resistance \\
\hline BG-11 plus 1 fold of K2HPO4 salt to Leptolyngbya sp.2 & $0.193 \pm 0.003$ & $4.32 \pm 0.22$ & Resistance \\
\hline BG-110plus 1 fold of K2HPO4 salt to Leptolyngbya sp.2 & $0.098 \pm 0.004$ & $6.25 \pm 0.18$ & $9 \pm 1.09$ \\
\hline No K2HPO4 salt BG-11 to Leptolyngbya sp.2 & No growth & No growth & $15 \pm 0.89$ \\
\hline BG-11plus 0.5 fold of NaNO3 salt to Leptolyngbya sp.1 & $0.169 \pm 0.003$ & $7.37 \pm 0.9$ & Resistance \\
\hline BG-11 plus 1 fold of NaNO3 salt to Leptolyngbya sp.1 & $0.194 \pm 0.008$ & $8.01 \pm 0.1$ & Resistance \\
\hline BG-11plus 0.5 fold of NaNO3 salt to Leptolyngbya sp.2 & $0.157 \pm 0.005$ & $4.8 \pm 0.24$ & Resistance \\
\hline BG-11 plus 1 fold of NaNO3 salt to Leptolyngbya sp.2 & $0.168 \pm 0.006$ & $5.6 \pm 0.17$ & Resistance \\
\hline BG-11plus 0.5 fold of Ferric ammonium citrate to Leptolyngbya sp.1 & $0.131 \pm 0.001$ & $6.08 \pm 0.79$ & Resistance \\
\hline BG-11 plus 1 fold of Ferric ammonium citrate to Leptolyngbya sp.1 & $0.155 \pm 0.003$ & $5.44 \pm 0.18$ & Resistance \\
\hline No Ferric ammonium citrate BG-11 to Leptolyngbya sp.1 & $0.148 \pm 0.005$ & $5.44 \pm 0.14$ & Resistance \\
\hline BG-11plus 0.5 fold of Ferric ammonium citrate to Leptolyngbya sp.2 & $0.13 \pm 0.001$ & $3.04 \pm 0.58$ & Resistance \\
\hline BG-11 plus 1 fold of Ferric ammonium citrateto Leptolyngbya sp.2 & $0.179 \pm 0.006$ & $3.04 \pm 0.35$ & Resistance \\
\hline No Ferric ammonium citrate BG-11 to Leptolyngbya sp.2 & $0.145 \pm 0.002$ & $1.76 \pm 0.9$ & Resistance \\
\hline BG-11plus 0.5 fold of CuSO4.5H2O salt to Leptolyngbya sp.1 & No growth & No growth & $14 \pm 0$ \\
\hline BG-11 plus 1 fold of CuSO4. $5 \mathrm{H} 2 \mathrm{O}$ salt to Leptolyngbya sp.2 & No growth & No growth & $10 \pm 1.26$ \\
\hline BG-11plus 0.5 fold of CuSO4.5H2O salt to Leptolyngbya sp.1 & No growth & No growth & $11 \pm 0$ \\
\hline BG-11 plus 1 fold of CuSO4. 5H2O salt to Leptolyngbya sp.2 & No growth & No growth & $9.5 \pm 0$ \\
\hline BG-11 plus 0.5 fold of MgSO4. 7 H2O salt to Leptolyngbya sp.1 & $0.144 \pm 0.003$ & $4.16 \pm 0.33$ & $9 \pm 0.63$ \\
\hline BG-11 plus 1 fold of MgSO4. 7H2O salt to Leptolyngbya sp.1 & $0.184 \pm 0.005$ & $4.807 \pm 0.1$ & $7.5 \pm 0.37$ \\
\hline BG-11 plus 0.5fold of MgSO4. 7H2O salt to Leptolyngbya sp.2 & $0.133 \pm 0.008$ & $2.4 \pm 0.11$ & $8 \pm 0$ \\
\hline BG-11 plus 1 fold of MgSO.4. 7H2O salt to Leptolyngbya sp.2 & $0.188 \pm 0.004$ & $3.044 \pm 0.98$ & $7.5 \pm 0.31$ \\
\hline BG-11 plus 0.5 fold of micronutrients [A5+CO] to Leptolyngbya sp.1 & $0.118 \pm 0.001$ & $2.24 \pm 0.8$ & Resistance \\
\hline BG-11 plus 1 fold of micronutrients [A5+ CO] to Leptolyngbya sp.1 & $0.13 \pm 0.005$ & $1.76 \pm 0.19$ & Resistance \\
\hline BG-11 plus 0.5 fold of micronutrients [A5+CO] to Leptolyngbya sp.2 & Cell death & Cell death & - \\
\hline BG-11 plus 1 fold of micronutrients [A5+CO] to Leptolyngbya sp.2 & Cell death & Cell death & - \\
\hline Routine BG-11 (Control) to Leptolyngbya sp.1 & $0.135 \pm 0.004$ & $2.24 \pm 0.28$ & $8 \pm 0.7$ \\
\hline Routine BG-11 (Control) to Leptolyngbya sp.2 & $0.13 \pm 0.001$ & $1.76 \pm 0.37$ & $7.5 \pm 0.31$ \\
\hline
\end{tabular}

Table 2 shows that combination of antibiotics and extracts had better results than their single use. According to the first row of this table, MIC of the extract alone is 80 $\mu \mathrm{g} / \mathrm{mL}$; while MIC in combination with antibiotic has decreased to $20 \mu \mathrm{g} / \mathrm{mL}$. MIC for antibiotic alone in this case is $1.2 \mu \mathrm{g} / \mathrm{mL}$, while in combination with the extract it decreased to $0.3 \mu \mathrm{g} / \mathrm{ml}$. The results demonstrated that the extracts combined with antibiotics significantly increased antimicrobial inhibition effects. Biomass increased with increasing concentrations of NaNO3, K2HPO4, Ferric ammonium citrate in $\mu$ max and productivity tests were considerable ( 24 ). Table 2 shows the importance of salts, particularly phosphate salt in the BG-11 culture medium for biomass production and growth of Leptolyngbya $s p$. This is probably applicable to all Cyanobacteria. K2HPO4 is so important that its absence is quite clear.

The importance of phosphorus and potassium content along with the buffering capacity of K2HPO4 indicate that Cyanobacteria will not be able to grow in the absence of K2HPO4. This condition is also true with the lower concentration of nitrogen in the culture medium. The nitrogen limited BG-110 culture media was used. Yellowish pigments produced in this medium show a major reduction in $\mu$ max with chlorophyll-a test calculation. Contrary to this phenomenon, higher values in $\mu$ max test were observed as a result of high $\mathrm{MgSO} 4$ concentration showing 
increased chlorophyll-a pigment. Absence of growth at two-fold increased micronutrients solution (A5+CO) indicates toxic effect of the higher concentrations of the trace elements. Increased antimicrobial activity at higher concentration of MgSO4, could be attributed to its sulfur content (25).

Importance of phosphorus, and nitrogen for cyanobacteria growth is in agreement with the reports by Downing et al. (26), Reynolds (27), Rolland et al. (28), and Xie et al. (29). Increased concentration of copper salt and micronutrients solution (A5+CO) resulted in no growth promotion confirming Ruiter et al. work (30). According to antimicrobial test results, Leptolyngbya species have more antimicrobial effects against the Gram positive bacteria and Candida genera than the Gram negative or fungi; this could be due to hydrophobic property of Gramnegative bacteria outer membrane lipopolysaccharides. The results indicate that the increased antimicrobial activity and biomass production are reversely related.

\section{Acknowledgements}

None Declared.

\section{Authors' Contribution}

None Declared.

\section{Financial Disclosure}

None Declared.

\section{Funding/Support}

None Declared.

\section{References}

1. Ejim L, Farha MA, Falconer SB, Wildenhain J, Coombes BK, Tyers $\mathrm{M}$, et al. Combinations of antibiotics and nonantibiotic drugs enhance antimicrobial efficacy. Nat Chem Biol. 2011;7(6):348-50.

2. Kulik MartinM. The potential for using cyanobacteria (bluegreen algae) and algae in the biological control of plant pathogenic bacteria and fungi. Eur J Plant Pathol.1995;101(6):585-599.

3. Ozdemir G, Karabay NU, Dalay MC, Pazarbasi B. Antibacterial activity of volatile component and various extracts of Spirulina platensis. Phytother Res. 2004;18(9):754-7.

4. Shanab SANAA M M. Bioactive allelo-chemical compounds from Oscillatoria species (Egyptian isolates). Int J Agric Biol. 2007;9(4):617-621.

5. Douterelo I, Perona E, Mateo P. Use of cyanobacteria to assess water quality in running waters. Environ Pollut. 2004;127(3):377-84.

6. Mateo Pilar, Douterelo Isabel, Berrendero Esther, Perona Elvira. Physiological differences between two species of cyanobacteria in relation to phosphorus. J Phycol. 2006;42(1):61-66.

7. Perona E, Bonilla I, Mateo P. Use of benthic cyanobacteria to monitor water quality in a Spanish river. Use of Algae for Monitoring Rivers III. Agence de l'Eau Artois-Picardie, Douai. 1999:216-223.

8. Rippka Rosmarie, Deruelles Josette, Waterbury John B, Herdman Michael, Stanier Roger Y. Generic assignments, strain histories and properties of pure cultures of cyanobacteria.J General Microbiol. 1979;111(1):1-61.

9. Casamatta Dale A, Johansen Jeffrey R, Vis Morgan L, Broadwater Sharon T. Molecular and morphological characterization of ten polar and near - polar strains within the Oscillatoriales (Cyanobacteria). J Phycol. 2005;41(2):421-438.

10. Abed RM, Garcia-Pichel F, Hernandez-Marine M. Polyphasic characterization of benthic, moderately halophilic, moderately thermophilic cyanobacteria with very thin trichomes and the proposal of Halomicronema excentricum gen. nov., sp. nov. Arch Microbiol. 2002;177(5):361-70.

11. Chatchawan T, Peerapornpisal Y, Komárek Jiří. Diversity of cyanobacteria in man-made solar saltern, Petchaburi Province, Thailand-a pilot study. Fottea. 2011;11(1):203-214.

12. Joseph S. Ph. D thesis, Cochin University of Science and Technology, Kochi, India; 2005.

13. van Baalen Chase. Further observation on growth of single cells of coccoid blue-green algae. Journal of Phycology. 1967;3(3):154-157.

14. Danielson T. Protocols for Collecting Water Grab Samples in Rivers, Streams, and Freshwater Wetlands. 2004.

15. Waterbury JohnB, Dworkin Martin, Falkow Stanley, Rosenberg Eugene, Schleifer Karl-Heinz, Stackebrandt Erko. The Cyanobacteria-Isolation, Purification and Identification. In: Waterbury JohnB, Dworkin Martin, Falkow Stanley, Rosenberg Eugene, Schleifer Karl-Heinz, Stackebrandt Erko, editors.The Prokaryotes.Springer US; 2006. p. 1053-1073.

16. Margheri Maria Cristina, Bosco Marco, Giovannetti Luciana, Ventura Stefano. Assessment of the genetic diversity of halotolerant coccoid cyanobacteria using amplified 16S rDNA restriction analysis. FEMS Microbiol Lett. 1999;173(1):9-16.

17. Van Run Jaap, Cohen Yehuda. Ecophysiology of the Cyanobacterium Dactylococcopsis salina: Effect of Light Intensity, Sulphide and Temperature. J Gen Microbiol.1983;129(6):1849-1856.

18. Challouf Rafika, Trabelsi Lamia, Ben Dhieb Rym, El Abed Omeya, Yahia Ali, Ghozzi Khamissa, et al. Evaluation of cytotoxicity and biological activities in extracellular polysaccharides released by cyanobacterium Arthrospira platensis. Brazil Arch Biol Technol. 2011;54(4):831-838.

19. Silveira Carolina Pereira, Torres-Rodríguez Josep M, AlvaradoRamírez Eidi, Murciano-Gonzalo Francisca, Dolande Maribel, Panizo Mercedes, et al. MICs and minimum fungicidal concentrations of amphotericin B, itraconazole, posaconazole and terbinafine in Sporothrix schenckii. J Med Microbiol. 2009;58(12):16071610.

20. Kamatou GPP, Viljoen AM, van Vuuren SF, van Zyl RL. In vitro evidence of antimicrobial synergy between Salvia chamelaeagnea and Leonotis leonurus. South Afr J Botany. 2006;72(4):634-636.

21. Suhail S, Biswas D, Farooqui A, Arif JM, Zeeshan M. Antibacterial and free radical scavenging potential of some cyanobacterial strains and their growth characteristics. J Chem Pharm Res. 2011;3(2):472-478.

22. El-Baky Hanaa H Abd, El Baz Farouk K, El-Baroty Gamal S. Production of phenolic compounds from Spirulina maxima microalgae and its protective effects in vitro toward hepatotoxicity model. Afr J Pharm Pharmacol. 2009;3(4):133-139.

23. Dixon RA, Al-Nazawi M, Alderson G. Permeabilizing effects of subinhibitory concentrations of microcystin on the growth of Escherichia coli. FEMS Microbiol Lett. 2004;230(2):167-70.

24. Raghuvanshi Ruchika, Singh Surendra, Bisen Prakash Singh. Iron mediated regulation of growth and siderophore production in a diazotrophic cyanobacterium Anabaena cylindrical. Indian J Exp Biol. 2007;45(6):563.

25. Rania MA, Hala MT. Antibacterial and antifungal activity of cyanobacteria and green microalgae. Evaluation of medium components by placket-burman design for antimicrobial activity of Spirulina platensis. Global J Biotechnol Biochem. 2008;3(1):22-31.

26. Downing John A, Watson Susan B, McCauley Edward. Predicting cyanobacteria dominance in lakes. Canadian J Fisheries Aquatic Sci. 2001;58(10):1905-1908.

27. Reynolds CS. Non-determinism to probability, or N: P in the community ecology of phytoplankton: Nutrient ratios. Archiv für Hydrobiologie. 1999;146(1):23-35.

28. Rolland Anne, Bird David F, Giani Alessandra. Seasonal changes in composition of the cyanobacterial community and the occurrence of hepatotoxic blooms in the eastern townships, Quebec, 
Canada.J Plankton Res. 2005;27(7):683-694.

29. Xie L, Xie P, Li S, Tang H, Liu H. The low TN:TP ratio, a cause or a result of Microcystis blooms? Water Res. 2003;37(9):2073-80.

30. Rueter John G, Petersen Richard R. Micronutrient effects on cyanobacterial growth and physiology. New Zealand J Marine Freshwater Res. 1987;21(3):435-445.
Please cite this paper as: Abazari M, Zarrini G, Rasooli I. Antimicrobial Potentials of Leptolyngbya sp . and Its Synergistic Effects With Antibiotics. Jundishapur J Microbiol. 2013; 6(4): e6536. DOI: 10.5812/ jjm.6536 